\title{
Lossless equalization of frequency combs
}

\author{
Víctor Torres-Company ${ }^{1, *}$, Jesús Lancis $^{1}$, and Pedro Andrés ${ }^{2}$ \\ ${ }^{1}$ GROC-UJI, Departament de Física, Universitat Jaume I, 12071 Castelló, Spain \\ ${ }^{2}$ Departamento de Óptica, Universidad de Valencia, 46100 Burjasot, Spain \\ *Corresponding author: vtorres@fca.uji.es
}

\begin{abstract}
Compiled April 29, 2008
Frequency combs obtained by sinusoidal phase modulation of narrow-band continuous-wave lasers are widely used in the field of optical communications. However, the resulting spectral envelope of the comb is not flat. In this Letter, we propose a general and efficient approach to achieve flat frequency combs with tunable bandwidth. The idea is based on a two-step process. First, efficient generation of a train with temporal flat-top-pulse profile is required. Second, we use large parabolic phase modulation in every train period in order to map the temporal intensity shape into the spectral domain. In this way, the resulting spectral envelope is flat and the size is tunable with the chirping rate. Two different schemes are proposed and verified through numerical simulations.

(C) 2008 Optical Society of America

OCIS codes: $060.0060,070.1170,060.5060,230.2090$
\end{abstract}

Stable, equally spaced frequency combs have lead to several applications in optical communications, such as high-fidelity short-light-pulse generation [1], arbitrary radio-frequency ( $\mathrm{RF}$ ) waveform generation [2], and densed wavelength division multiplexing [3]. For these applications, the frequency comb is usually generated by sinusoidal phase modulation of a continuous-wave (CW) narrow-band laser as it provides high stability, frequency spacing tunability and large bandwidth with a relatively low-amplitude RF driving signal. However, the spectral envelope modulating the comb is not flat, which may limit some of the above applications.

In the last years, several efficient solutions have been proposed and demonstrated to achieve a high degree of flatness over a specific spectral region in the comb. We mention, e.g., the optimization of dual-drive MachZehnder modulators $[4,5]$; concatenation of two electrooptic modulators $[6,7]$; or mixing of two sinusoidal $\mathrm{RF}$ signals in a single electro-optic phase modulator (EOPM) [8]. In these schemes, the spectral flatness is achieved by an accurate optimization of the physical parameters of the modulators, i.e., modulation indexes, bias voltages, and phase shifters. With current technology in $\mathrm{LiNbO}_{3}$ modulators, it is possible to implement some of the solutions obtained from numerical investigations. However, the achieved bandwidth of the frequency comb results difficult to tune, given a set of optimization parameters.

In this Letter, we propose an alternative and general method to achieve bandwidth tunable, ultra-flat frequency combs. It is based on the generation of flattop pulses in the time domain and subsequent time-tofrequency (TTF) conversion. Let us remind that TTF converters are devices implemented by concatenating a group-delay dispersive (GDD) medium and a time lens, satisfying a specific relation between the GDD amount and the chirping rate [9]. Overall, these photonic devices have the inherent capability of mapping the intensity of a single pulse into the spectral domain. Therefore, the energy spectrum of the signal at the output corresponds to a scaled version of the temporal intensity of the input signal. The scaling factor is just given by the chirping rate of the time lens. For the purposes of this Letter, it is important to recognize that a simplified configuration of this setup has been recently reported by Azaña et al., where the dispersive element can be dropped [10]. Following their work, since the action of a time lens can be mathematically described as a multiplicative element impressing parabolic phase modulation in the time domain, $\exp \left(i K t^{2} / 2\right)$, where $K$ denotes the chirping rate, TTF conversion is achieved whenever the following inequality is satisfied

$$
|K|>>\frac{\Delta \omega^{2}}{4 \pi} .
$$

Here, $\Delta \omega$ denotes the spectral bandwidth of the pulse at the input. This situation corresponds to the dual situation, in the spectral domain, of the Fraunhofer far-field effect [10]. In other words, large temporal modulation on a pulse also induces TTF conversion. Conventionally, these devices have been used for measuring the intensity distribution of ultrashort pulses with common optical spectrum analyzers $[9,10]$. In this Letter, we propose their use as frequency comb flatteners.

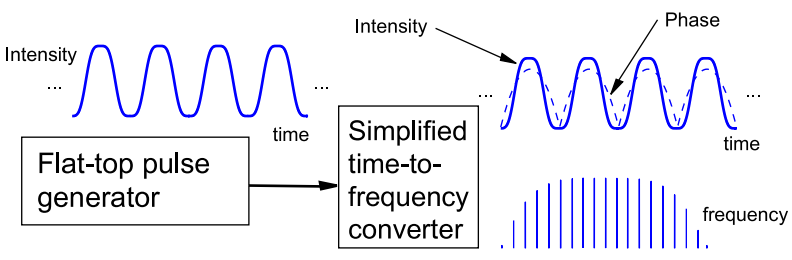

Fig. 1. General scheme for achieving flat-top frequency combs based on TTF conversion.

Our general setup is depicted in Fig. 1. Let us assume we have an optical source providing a train of flat-top- 
intensity pulses, $I_{\text {in }}(t)=\sum_{n} I_{0}(t-n T)$, where $I_{0}(t)$ denotes the intensity profile of the elementary pulse, and $T$ the period. We shall clarify later how we can achieve flat-top pulses by means of sinusoidal phase modulation. Now we consider that this train is inserted into a device impressing parabolic phase modulation on every single pulse constituting the train, i.e., a periodic time lens. Thus, accurate synchronization between both sequences is required. The key is to note that if the time lens posses a chirping rate satisfying the inequality expressed by Eq. (1), the output energy spectrum of every elementary pulse will be a scaled version of the input intensity $I_{0}(t)$. Since this TTF conversion acts on every pulse, the output energy spectrum will be given by

$$
S(\omega)=I_{0}(\omega / K) \sum_{n=-\infty}^{\infty} \delta(\omega-2 \pi n / T),
$$

where $\omega$ represents the angular frequency referred to the optical carrier. This equation indicates that flat frequency combs can be achieved provided that we have a proper source of flat-top intensity pulses and a repetitive time lens satisfying the TTF conversion condition. It is important to note that the spectral bandwidth of the envelope of the comb is tunable with $K$. Different chirping rates do not change the shape, only the scale of the envelope. Thus, we can estimate the effective number of spectral lines as

$$
n \sim \frac{|K| \sigma}{2 \pi f_{r}},
$$

where $\sigma$ is the temporal duration of $I_{0}(t)$ and $f_{r}=T^{-1}$. Larger $K$ parameters lead to broader frequency combs. We have then reduced the problem of generating flat frequency combs to the generation of flat-top-intensitypulse trains.

We should emphasize that, in principle, the original configuration of TTF converters [9] could be applied to this problem too. However, the required GDD circuit leads to a broadening of the input flat-top pulse before entering the time lens. This broadening may distort the TTF mapping because, as is well-known, time lenses are subject to temporal aberrations, specially when they are implemented with EOPMs [11]. Therefore, we prefer to restrict ourselves to the simplified version reported in [10].

At this stage, we must clarify the content of the flattop pulse generator box in Fig. 1. Although there are many different approaches in the literature reporting generation of flat-top pulse profiles, we concentrate on two examples that are quite efficient in terms of energy losses. The first one consists on the insertion of an EOPM in one arm of a Mach-Zehnder interferometer, which has a CW laser as input light, as sketched in Fig. 2(a). If we drive the EOPM with a sinusoidal RF signal, the optical complex field envelope at the output of the interferometer can be written as

$$
e(t)=A_{0}\left\{1+\exp (i \Phi) \exp \left[i \Delta \theta_{1} \sin \left(2 \pi f_{r 1} t\right)\right]\right\}
$$
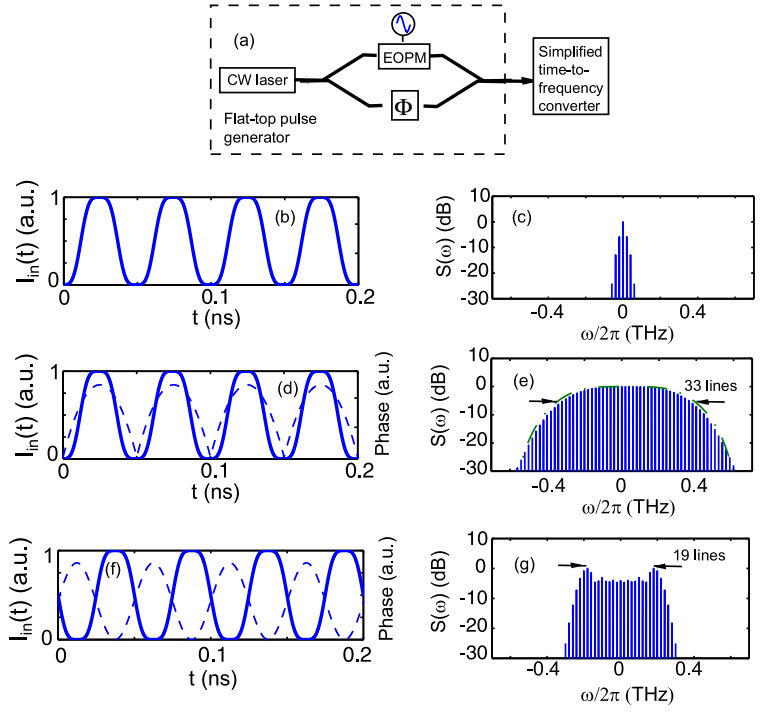

Fig. 2. (a) First proposal for flat-top comb generator; (b) intensity flat-top-pulse train; (c) corresponding normalized energy spectrum before entering the TTF converter; (d) intensity pulse train (solid line) modulated with an ideal periodic time lens (dashed line); (e) corresponding normalized frequency comb; (f) flat-top pulse train (solid line) phase-modulated with an EOPM driven with a sinusoidal signal (dashed line) featuring a periodic time lens satisfying TTF conversion; (g) corresponding normalized frequency comb. Numerical settings are explained in the text.

where $\Delta \theta_{1}$ denotes the modulation index, $f_{r 1}$ is the frequency of the RF signal, $\Phi$ is a constant related with the bias voltage, and $A_{0}$ is a constant irrelevant for our purposes. Since, $\Phi$ can be controlled with the bias voltage, we can set independently $\Phi=\pi / 2$ and $\Delta \theta_{1}=\pi / 2$. In this case, the corresponding light intensity profile and energy spectrum are plotted in Figs. 2(b) and (c), respectively, for $f_{r 1}=20 \mathrm{GHz}$. The spectral content consists on a series of equally spaced components whose weights are related to the Bessel function of the first kind. But thanks to the proper choice of the settings parameters, the pulse sequence in the time domain is formed by relatively flat-top pulses with a duty cycle $\sim 50 \%$. Thus, according to our theory, the comb can be equalized if, as sketched in Fig. 2(a), we concatenate a simplified TTF converter, i.e., a repetitive time lens with a chirping rate satisfying Eq. (1). Figures 2(d) and (e) plot in the time and spectral domain, respectively, the numerical result for an ideal (parabolic) time lens acting on every single pulse with a chirping rate of $K=-0.158 \mathrm{ps}^{-2}$. For completeness, the ideal case given by $I_{0}(\omega / K)$ is plotted in dash-dotted line in Fig. 2(e). The TTF mapping is evident, and the flatness of the comb excellent. However, parabolic phase modulation is difficult to implement. By relaxing this condition and assuming that parabolic time lenses are implemented with an EOPM with the same chirping rate and at the same driving frequency 
(which, since $K=-4 \pi^{2} \Delta \theta f_{r_{1}}^{2}$ for an EOPM, this would be achieved with a modulation index $\Delta \theta=10 \mathrm{rad}$ ), the comb should be as the one in Fig. 2(g). We observe a decreasing of the achieved spectral bandwidth and spectral ripples in the outer frequencies. These features are due to the temporal aberrations introduced by the imperfect time lens. Temporal aberrations are notable because of the high duty cycle and the long rise and fall times of the pulse inside a period. However, we note that even in this case, the flatness region of the achieved comb can be increased linearly with the chirping rate $K$. In a practical realization, the same $\mathrm{RF}$ source could be used to drive both EOPMs, and the different modulation indexes could be achieved with an RF amplifier before the second EOPM. In that case, not only the bandwidth but also the line spacing become tunable through the driving RF signal.
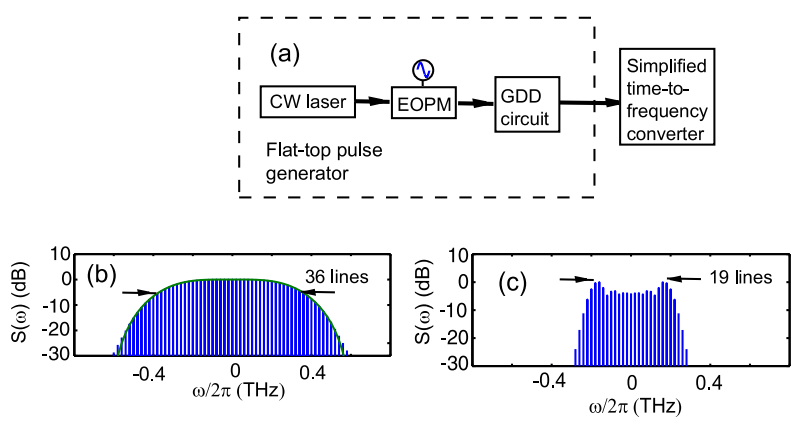

Fig. 3. (a) Second proposal for flat-top comb generator; (b)normalized frequency comb achieved when a perfect periodic time lens is used as TTF converter; (c) achieved normalized frequency comb when an EOPM is used as time lens. Numerical settings are explained in the text.

Our next example is sketched in Fig. 3. A CW laser is phase-modulated with an EOPM driven with a sinusoidal signal with frequency $f_{r 2}$. Then, the light is inserted in a GDD medium, e.g., a single-mode fiber for simplicity, satisfying $\beta_{2} z=\left(4 \pi f_{r 2}^{2}\right)^{-1}$, where $\beta_{2}$ is the group velocity dispersion coefficient of the fiber and $z$ the corresponding length. In this case, the output complex field may be written as [12]

$$
e(t)=A_{0}^{\prime} \exp [-i V(t)]\{1+i \exp [2 i V(t)]\}
$$

where $V(t)=\Delta \theta_{2} \sin \left(2 \pi f_{r 2} t\right), \Delta \theta_{2}$ is the modulation index, and $A_{0}^{\prime}$ another irrelevant constant. Comparing Eq. (5) with Eq. (4), one notes that a flat-top intensity profile can also be achieved whenever $\Delta \theta_{2}=\pi / 4$ [13]. The energy spectrum consists on a nonuniform frequency comb. However, as sketched in Fig. 3(a), by concatenating a simplified TTF converter, a flat bandwidth-tunable frequency comb can be achieved. Figure 3(b) shows the energy spectrum when ideal parabolic phase modulation is implemented in every period with the same chirping rate as for the case of Fig. 2(e). Figure 3(c) represents the more realistic case when the simplified TTF converter is implemented with an EOPM. Again, due to the $~ 50 \%$ duty cycle, temporal aberrations play a significant role, leading to the spectral bandwidth decreasing and distortion. We must emphasize that this last result has been successfully reported by Yamamoto et al. [14]. But there, the relation with the generalized TTF conversion developed in this work was not pointed out. We point out that Eq. (3) agrees with the observed linear dependence of the spectral bandwidth with the modulation index.

In conclusion, a new technique to achieve tunable flattop frequency combs is proposed. It consists on applying a repetitive time lens on a flat-top-pulse train so that it induces TTF conversion in every single period. When the flat-top-pulse train is implemented with phase-only modulation, our approach constitutes a lossless spectral equalizer. Two different schemes are presented. Our numerical simulations show that they are realizable with current technology.

This work was supported by Dirección General de Investigación Cientifica y Técnica, Spain and Fondos Europeos para el Desarrollo Regional, under projects FIS2007-62217 and SAUUL (Science and Applications of Ultrafast Ultraintense Lasers) in the program CONSOLIDER. V. Torres thanks Ministerio de Educación y Ciencia for a Formación Profesorado Universitario fellowship.

\section{References}

1. Z. Jiang, C. B. Huang, D. E. Leaird, and A. M. Weiner, Nature Photon. 1, 463 (2007).

2. P. J. Delfyett, S. Gee, M. T. Choi, H. Izadpanah, W. Lee, S. Ozharar, F. Quinlan, and T. Yilmaz, J. Lightwave Technol. 24, 2701 (2006).

3. T. Ohara, H. Takara, T. Yamamoto, H. Masuda, T. Morioka, M. Abe, and H. Takahashi, J. Lightwave Technol. 24, 2311 (2006).

4. T. Sakamoto, T. Kawanishi, and M. Izutsu, Opt. Lett. 32, 1515 (2007).

5. I. L. Gheorma and G. K. Gopalakrishnan, IEEE Photon. Technol. Lett. 19, 1011 (2007).

6. M. Fujiwara, M. Teshima, J. Kani, H. Suzuki, N. Takachio, and K. Iwatsuki, J. Lightwave Technol. 21, 2705 (2003).

7. T. Healy, F. C. G. Gunning, A. D. Ellis, and J. D. Bull, Opt. Express 15, 2981 (2007).

8. S. Ozharar, F. Quinlan, I. Ozdur, S. Gee, and P. J. Delfyett, IEEE Photon. Technol. Lett. 20, 36 (2008).

9. M. T. Kauffman, W. C. Banyai, A. A. Godil, and D. M. Bloom, Appl. Phys. Lett. 64, 270 (1994).

10. J. Azaña, N. K. Berger, B. Levit, and B. Fischer, IEEE Photon. Technol. Lett. 16, 882 (2004).

11. C. V. Bennett and B. H. Kolner, IEEE J. Quantum Electron. 37, 20 (2001).

12. V. Torres-Company, J. Lancis, and P. Andrés, Opt. Express 14, 3171 (2006).

13. T. Komukai, T. Yamamoto, and S. Kawanishi, IEEE Photon. Technol. Lett. 17, 1746 (2005).

14. T. Yamamoto, T. Komukai, K. Takada, and A. Susuki, Electron. Lett. 43, 1040 (2007). 